\title{
Review of: "Establishment of a cardiac telehealth program to support cardiovascular diagnosis and care in a remote, resource-poor setting in Uganda"
}

Joselyn Rwebembera ${ }^{1}$, Bruno Nascimento ${ }^{2}$

1 Uganda Heart Institute

2 Clinic Hospital UFMG

Potential competing interests: The author(s) declared that no potential competing interests exist.

\section{Bridging the Great Divide: Can Telemedicine Tackle Access to Cardiac Care in Africa?}

Developing countries need telemedicine applications that help in many situations, when physicians are a small number in respect to the population, when specialized physicians are scarce and centered in capital cities, when transport systems are challenging, among many other constraints. ${ }^{1}$ In such under-resourced settings, telemedicine has the potential to act as the 'safety net' that allows many underprivileged peoples an access to state-of-the-art medical care by accessing the few, highly specialized personnel who would otherwise not be within their reach. ${ }^{2}$ However the settings where telemedicine is expected to be most beneficial in closing health care delivery gaps are the same which naturally face the highest barriers to telehealth implementation.

The use of long distance communications for medical purposes extends into antiquity. ${ }^{3}$ For example, medicine "in absentia" was practiced in the 17th century common era as patients sent urine samples to distant physicians who, in turn, provided diagnoses based on uroscopy charts patterned after those used by the ancient Greeks. ${ }^{4}$ In return, local physicians and their patients received a "prescription-by-post" containing detailed instructions on regimen. ${ }^{5}$ Anecdotal justification for use of the telephone in distant diagnosis can be found in an early report in The Lancet in 1879. A child was suspected of having "croup". The physician telephoned the mother and asked her to "lift the child to the telephone and let me hear it cough." Subsequently, he declared: "That is not the croup". ${ }^{6}$ Einthoven is credited with the first use of the prefix "tele" in a medical context that appeared in an article published in $1906 .{ }^{7}$ Following several applications, telemedicine came of age during the 1970s. The first prototype telemedicine program was established in 1968 in Boston, linking the Medical Station at Logan International Airport with Massachusetts General Hospital. Today, telemedicine can be found almost in every country in the world, albeit at highly variable stages of development.

There is a myriad of current telemedicine definitions in the literature. The World Health Organization in 
2009 counted 104 distinct, peer-reviewed definitions of the concept. ${ }^{2}$ Generally, telemedicine refers to the use of electronic communications and information technology to provide medical care at a distance. Operationally, it covers 2 broad aspects: 1) Virtual interaction between a patient and a health care worker; 2) The flow of information. There are also variable types of telemedicine visits, broadly classified as synchronous or asynchronous, each class having further sub-divisions under it. ${ }^{8}$

Globally, virtual care has offered a lifeline during a global health crisis. Covid-19 has accelerated the adoption of telemedicine globally, ${ }^{9}$ pushing its fast emergence as one of the most defining trends of this decade. The platform's sudden growth and swift evolution of the landscape could have permanent implications, ${ }^{10}$ and developing countries are not spared.

Even in developed and middle income countries where telemedicine has existed for a long time, the COVID-19 pandemic has given it a whole new and enviable face. ${ }^{9}$ During the challenging times of the pandemic, objective results of telemedicine for multiple applications have been reported: from teleconsultations and telemonitoring - reducing the overload of collapsed health systems ${ }^{11}$ - to training of healthcare personnel and provision of remote interpretation of exams - increasing access to diagnosis whereas lowering the hazard of contamination. ${ }^{12}$ Concomitantly, the number of publications on the role and benefits of telehealth has skyrocketed in the past 2 years of the pandemic.

The requirements of a telemedicine program span 3 broad categories; 1) Hardware; 2) Software; and 3) Relevant knowledge and skills. Other unclassified requirements include access to electricity, legislative and, especially, regulatory oversight. With these listed requirements, not surprisingly - variations in digital preparation, maturity of health systems and hospitals, availability, access, and implementation of telemedicine are experienced across the world. ${ }^{13}$ Many aspects of telemedicine remain complex and contentious even in settings where the field is fairly advanced. For example, matters of billing, reimbursement and coverage remain a major challenge due to the lack of uniformity. ${ }^{14}$ Also, patient information privacy and confidentiality remains a contentious area in telemedicine practice that is not unique to developed countries. ${ }^{9}$

Nonetheless, the wait for solutions to these and other LMIC-specific barriers ${ }^{1} 2$ should not impede innovative efforts to the application of a potentially bridging platform in settings where the health service gap is widest. In such a bid - to address workforce shortages and expand access to care, Alyssa and colleagues $^{15}$ developed a telemedicine program incorporating existing infrastructure for delivery of cardiovascular care in a remote setting in Northern Uganda. They employed electronic flow of information, but without virtual interaction between patients and the distant cardiologists. Such a modification of 'full telemedicine' is an innovative way to circumvent the patient-end telemedicine barriers, such as patient access to the required hardware, software, and service usage literacy. This study had a strong advantage 
of a highly competent nurse who took history and basic aspects of physical examination, conducted electrocardiograms and echocardiograms followed by transmission of these to distant capital city-based cardiologists who synthesized a diagnosis and care plan based on the available history, physical exam and imaging findings. Indeed, the authors found a significant burden of cardiac disease, medical care plans were outlined, and qualitative analysis revealed high levels of acceptance and appreciation, citing cost and time saving benefits. Although there was no usual care comparison group in this study, the authors do prove a feasibility case for (cardiac) telemedicine programs in LMICs.

However, outside of a research study setting, the conduction of such a program would face multiple barriers and challenges in Uganda, almost with non-take-off implications. A recent public health facility based survey in Uganda that assessed readiness to integrate telemedicine at public health facilities in the country found several factors that might hamper the adoption and integration of the service. ${ }^{16}$ These included low levels of awareness of telemedicine among health workers and even lower levels among patients, unstable internet and electricity services, high cost of electronic devices, and low computer literacy especially among patients. ${ }^{16}$

From our local experience in Uganda, a variety of vendors providing general and specialized telemedicine technology and services have sprouted in the country following the COVID pandemic pressure, albeit the majority, if not all, being private providers. If left entirely to the private sector, there is likely going to be exploitation of patients regarding the levied fees, and exclusion of the population that is unable to afford the services. Governments and health ministries in LMICs such as Uganda need to embrace and invest in the inevitable development and requirement of telehealth to ensure equitable access.

Several population indices speak to the possibility of telemedicine in resource constrained countries. For example, in Sub-Saharan Africa, smartphone penetrance is expected to reach $66 \%$ by $2025 .{ }^{17}$ Suzuki et al. reported that in countries such as South Africa, Egypt, Morocco, and Algeria, high internet and mobile penetration rates open up the possibility for telemedicine. ${ }^{18} \mathrm{~A}$ systematic review of initiatives in Kenya showed that there were several eHealth projects, mostly focused on primary care and HIV/AIDS, but vast inequalities existed between more central and remote parts of the country in terms of delivery. ${ }^{19} \mathrm{~A}$ few LMIC settings have made even more tangible progress. For example, in Latin America, a prolific telemedicine network (a collaboration between public universities, the Ministry of Health and local boards of health, with co-funding by private and public grants) has been providing nation-wide access to tele-ECG, subsequentially expanding access to other modalities, such as acute coronary care, tele-retinography, tele-echocardiography/echo-screening and, recently, Covid-19 care. ${ }^{20}$ 
our patients are uniquely positioned to take advantage of and benefit from telemedicine. The international community, funders and partners in research and health systems improvement need to pay special attention to telemedicine in developing and under-developing nations, where the need is highest, while access and usability of technology are lowest. Regional frameworks that allow for collaboration and sharing or leveraging of resources need to be strengthened. The wide implementation of telemedicine especially more advanced modalities - also depend on deep regulatory discussions and adjustments, involving governments, stakeholders, class representatives and ultimately users. ${ }^{21}$ A frequent common sense that telemedicine could "replace" healthcare staff, or reduce the payment or reimbursement for health services also needs to be addressed.

Commitment to equal access to quality care to all and digital health is a critical enabler for the overall transformation of the digital health ecosystem. Barriers that are specific to telemedicine in LMICs, such as technology and internet-specific barriers, inadequate infrastructure and lack of digital literacy are major impediments in telemedicine implementation ${ }^{9}$ and require priority attention. Failure to acknowledge and address these factors can further increase the digital disparity and lack of care access to those who may need it the most. Further feasibility study, particularly the integration of telemedicine in public health systems could be one of the key steps to reducing the prevailing injustice of healthcare inequalities.

1. Combi C, Pozzani G and Pozzi G. Telemedicine for developing countries. Applied clinical informatics. 2016;7:1025-1050.

2. Ryu S. Telemedicine: opportunities and developments in member states: report on the second global survey on eHealth 2009 (global observatory for eHealth series, volume 2). Healthcare informatics research. 2012;18:153-155.

3. Bashshur R and Shannon GW. History of telemedicine: evolution, context, and transformation: Mary Ann Liebert New Rochelle, NY; 2009.

4. Fine LG. Circle of urine glasses: Art of uroscopy. American journal of nephrology. 1986;6:307-311.

5. Wild W. Doctor-patient correspondence in eighteenth-century Britain: A change in rhetoric and relationship. Studies in Eighteenth-Century Culture. 2000;29:47-64.

6. Bashshur RL, Shannon GW, Smith BR, Alverson DC, Antoniotti N, Barsan WG, Bashshur N, Brown EM, Coye MJ and Doarn CR. The empirical foundations of telemedicine interventions for chronic disease management. Telemedicine and e-Health. 2014;20:769-800.

7. Einthoven W. Het telecardiogram [The tele-cardiogram]. Ned T Geneesk. 1906;50:1517-1547.

8. Hare N, Bansal P, Bajowala SS, Abramson SL, Chervinskiy S, Corriel R, Hauswirth DW, Kakumanu 
S, Mehta R and Rashid Q. Work group report: COVID-19: Unmasking telemedicine. The Journal of Allergy and Clinical Immunology: In Practice. 2020;8:2461-2473. e3.

9. Bhaskar S, Bradley S, Chattu VK, Adisesh A, Nurtazina A, Kyrykbayeva S, Sakhamuri S, Yaya S, Sunil T and Thomas P. Telemedicine across the globe-position paper from the COVID-19 pandemic health system resilience PROGRAM (REPROGRAM) international consortium (Part 1). Frontiers in public health. 2020;8:644.

10. Mesko B. Health IT and digital health: The future of health technology is diverse. Journal of clinical and translational research. 2018;3:431.

11. Nascimento BR, Brant LC, Castro ACT, Froes LEV, Ribeiro ALP, Cruz LV, Araújo CB, Souza CF, Froes ET and Souza SD. Impact of a large-scale telemedicine network on emergency visits and hospital admissions during the coronavirus disease 2019 pandemic in Brazil: Data from the UNIMED-BH system. Journal of telemedicine and telecare. 2020:1357633X20969529.

12. Pimentel SLG, Nascimento BR, Franco J, Oliveira KKB, Fraga CL, Macedo FVBd, Raso LAdM, Ávila REd, Santos LPAd and Rocha RTL. Bedside echocardiography to predict mortality of COVID-19 patients beyond clinical data: Data from the PROVAR-COVID study. Revista da Sociedade Brasileira de Medicina Tropical. 2021;54.

13. Scott Kruse C, Karem P, Shifflett K, Vegi L, Ravi K and Brooks M. Evaluating barriers to adopting telemedicine worldwide: a systematic review. Journal of telemedicine and telecare. 2018;24:4-12.

14. Men J. Lack of reimbursement barrier to telehealth adoption. The American Journal of Managed Care. 2015.

15. DeWyer A, Scheel A, Kamarembo J, Akech R, Asiimwe A, Beaton A, Bobson B, Canales L, DeStigter $\mathrm{K}$ and Kazi DS. Establishment of a cardiac telehealth program to support cardiovascular diagnosis and care in a remote, resource-poor setting in Uganda. PloS one. 2021;16:e0255918.

16. Kiberu VM, Scott RE and Mars M. Assessing core, e-learning, clinical and technology readiness to integrate telemedicine at public health facilities in Uganda: a health facility-based survey. BMC health services research. 2019;19:1-11.

17. Holst C, Sukums F, Radovanovic D, Ngowi B, Noll J and Winkler AS. Sub-Saharan Africa-the new breeding ground for global digital health. The Lancet Digital Health. 2020;2:e160-e162.

18. Suzuki T, Hotta J, Kuwabara T, Yamashina H, Ishikawa T, Tani Y and Ogasawara K. Possibility of introducing telemedicine services in Asian and African countries. Health Policy and Technology. 2020;9:1322.

19. Njoroge M, Zurovac D, Ogara EA, Chuma J and Kirigia D. Assessing the feasibility of eHealth and mHealth: a systematic review and analysis of initiatives implemented in Kenya. BMC research notes. 2017;10:1-11.

20. Alkmim MB, Figueira RM, Marcolino MS, Cardoso CS, Abreu MPd, Cunha LR, Cunha DFd, Antunes AP, Resende AGdA and Resende ES. Improving patient access to specialized health care: the Telehealth Network of Minas Gerais, Brazil. Bulletin of the World Health Organization. 2012;90:373-378. 
21. Nittari G, Khuman R, Baldoni S, Pallotta G, Battineni G, Sirignano A, Amenta F and Ricci G. Telemedicine practice: review of the current ethical and legal challenges. Telemedicine and e-Health. 2020;26:1427-1437. 